\title{
Diretrizes Curriculares para os Cursos de Graduação em Geologia e Engenharia Geológica
}

L. Fantinel, V.A. Janasi, J.F.P. Assis, J.R. Alecrim, H.L.de Almeida, M. Compiani, R. Conceição, B.P. Duarte, G. Fauth, V.P. da Fonseca, P. Fortes, W.B. Leite Júnior, F. Mancini, M.G. de Menezes, C.H.da Silva, W. Silva Filho, E. Velloso, C.D.R Carneiro

\section{APRESENTAÇÃO}

O notável incremento, verificado nas últimas décadas, nas demandas sociais sobre o ambiente e sobre os recursos naturais, bem como as significativas mudanças na produção e transmissão do conhecimento científico e tecnológico tornaram inadiável a realização de amplo diagnóstico sobre a formação dos geólogos nas universidades brasileiras. Esse cenário permitiu que fossem organizadas duas reuniões sucessivas, denominadas de Seminário Nacional sobre Cursos de Graduação em Geologia, que contaram com apoio da Sociedade Brasileira de Geologia (SBGeo) e outras entidades. O I Seminário ocorreu em 2001, na cidade de Salvador (BA), e foi promovido pelo Instituto de Geociências da Universidade Federal da Bahia. O II Seminário ocorreu em 2002, na cidade de Campinas (SP), e foi promovido pelo Instituto de Geociências da Universidade Estadual de Campinas. O foco de ambos os encontros foi a análise dos rumos do ensino nos Cursos de Geologia no Brasil, tendo em vista o enfrentamento dos desafios que se apresentam no século XXI. Os resultados dos eventos foram sintetizados por Mesquita et. al. (2001), Sobreira (2005) e Carneiro (2005).

O II Seminário decidiu criar o Fórum Nacional de Cursos de Geologia, organismo permanente e representativo, do qual fazem parte todos os cursos de Geologia do país além de outras entidades, adiante listadas. Além disso, o encontro decidiu encaminhar ao Ministério da Educação e da Cultura (MEC) a proposta de Diretrizes Curriculares para cursos de Graduação em Geologia e Engenharia Geológica. A síntese, publicada por Nummer et al. (2005), reúne sugestões de alteração ao documento Diretrizes Curriculares para os Cursos de Geologia no País elaborado pela Comissão de especialistas no Ensino de Geologia e Oceanografia, designada pela Secretaria de Educação Superior do Ministério da Educação (SESU/MEC).

O documento assinado por Mesquita et al. (2001) fora encaminhado ao Presidente do Conselho Nacional de Educação (CNE) por meio do ofício $\mathrm{n}^{\circ}$ 108/01-GEO de 12.06.2001, que foi recebido no CNE em 26.06.2001 e transformado no EXPEDIENTE № 013245/2001-96 e encaminhado à Câmara de Educação Superior (CES), onde permaneceu até dezembro de 2001 com o Presidente da CES, Prof. Roberto Cláudio Frota Bezerra. Desde então, aguardase a designação de Comissão que deverá avaliar as sugestões e relatar, visando definir as Diretrizes Curriculares para os Cursos de Graduação de Geologia.

Em 2007, por ocasião do VII Encontro do Fórum, realizado nas cidades de São Paulo, Campinas e Rio Claro (SP), foi decidido alterar alguns pontos essenciais da proposta Diretrizes Curriculares para os Cursos de Geologia 
e Engenharia Geológica, anteriormente aprovada e publicada. O presente texto constitui a síntese do documento aprovado por unanimidade pelos representantes de 17 (dezessete) das 24 (vinte e quatro) universidades brasileiras que ministram o referido curso:

1. José Roberto Alecrim (Univ. do Amazonas UA)

2. Beatriz Paschoal Duarte (Univ. do Estado do Rio de Janeiro - UERJ)

3. Valdecir de Assis Janasi, Excelso Ruberti (Univ. de São Paulo - USP)

4. Mauricio Compiani (Univ. Estadual de Campinas - UNICAMP)

5. Washington B. Leite Júnior (Univ. Estadual Paulista - UNESP)

6. Harrizon Lima de Almeida (Univ. Federal da Bahia - UFBA-Barreiras);

7. Carlos Humberto da Silva (Univ. Federal de Mato Grosso-UFMT);

8. Lucia Maria Fantinel (Univ. Federal de Minas Gerais - UFMG);

10. Paulo Fortes (Univ. Federal do Espírito Santo - UFES);

11. Messias Gilmar de Menezes (Univ. Federal de Ouro Preto - UFOP);

12. Wellington Silva Filho (Univ. Federal do Ceará - UFCE);

13. José Fernando Pina Assis (Univ. Federal do Pará - UFPA);

14. Fernando Mancini (Univ. Federal do Paraná UFPR);

15. Emilio Velloso (Univ. Federal do Rio de Janeiro - UFRJ)

16. Vanildo Pereira da Fonseca (Univ. Federal do Rio Grande do Norte - UFRN);

17. Rommulo Conceição (Univ. Federal do Rio Grande do Sul -UFRGS);

18. Gérson Fauth (Univ. do Vale do Rio dos Sinos - UNISINOS);

Todo o processo de elaboração da proposta de diretrizes baseou-se em palestras ministradas por pesquisadores, professores universitários e contribuições de representantes da Sociedade Brasileira de Geologia (SBGeo), Conselho Federal de Engenharia, Arquitetura e Agronomia (CONFEA), Conselho Regional de Engenharia, Arquitetura e Agronomia de São Paulo (CREA-SP), Sindicato dos Geólogos no Estado de São Paulo (SIGESP) e Federação Nacional dos Geólogos (FEBRAGEO). O conjunto de temas compreendeu: diretrizes curriculares, conteúdo curricular, formação acadêmica, mercado de trabalho, desafios da Ciência contemporânea e a questão das licenciaturas.

O documento de Mesquita et al. (2001) constitui um substitutivo contendo destaques e discriminação de trechos modificados e/ou suprimidos, enquanto o texto publicado por Nummer et al. (2005) constitui proposta completa e atualizada de Diretrizes Curriculares para os Cursos de Graduação em Geologia e Engenharia Geológica. Uma vez que foram feitas mudanças naquele documento de 2001-2005, o presente documento reflete a posição formal do Fórum Nacional de Cursos de Geologia.

Campinas, maio de 2007.

Fórum Nacional de Cursos de Geologia.

\section{DIRETRIZES CURRICULARES PARA OS CURSOS DE GEOLOGIA E ENGENHARIA GEOLÓGICA}

\section{INTRODUÇÃO}

As Diretrizes Curriculares, conforme disposto no inciso II do artigo 53 da LDB - Lei No 9394/96 de 20 de dezembro de 1996, atendem ao espírito de flexibilização dos currículos de graduação. Pretende-se ultrapassar o atual modelo de currículos mínimos, que encerra elevado grau de detalhamento de disciplinas e cargas horárias e impede que sejam implementados projetos pedagógicos mais inovadores pelas Instituições de Ensino Superior (IES). As Diretrizes Curriculares diferem fundamentalmente, portanto, do modelo anterior de currículos mínimos.

A idéia fundamental que norteia o conceito de Diretrizes Curriculares refere-se à maior responsabilidade das IES, docentes, discentes e da sociedade, juntamente com o MEC, no objetivo de um ensino de graduação de qualidade e capaz de definir um diferencial na formação acadêmica e profissional de acordo com as necessidades de desenvolvimento do país.

Concebe-se assim, a graduação como uma etapa inicial de formação e não como um momento de esgotamento do conhecimento, considerando-se que, em uma Sociedade globalizada, na qual as mudanças no conhecimento são cada vez mais aceleradas, a chave para que o Ensino Superior acompanhe as transformações está na educação continuada.

Este aspecto dinâmico só é viável dentro de uma estrutura como a das Diretrizes Curriculares, que permitirá às IES definir diferentes perfis de seus 
egressos e adaptar tais perfis às rápidas mudanças do mundo moderno. Ou seja, estas Instituições terão liberdade para definir parte considerável de seus currículos.

A definição de perfis dos egressos de uma Instituição estará ligada à clara definição das capacidades criativas de cada uma delas, das responsabilidades e funções que os egressos poderão vir a exercer, dos problemas que serão capazes de resolver. Isso vai depender fundamentalmente da composição dos currículos plenos e das áreas de conhecimento que deverão contemplar em sua abrangência.

Os profissionais formados a partir das Diretrizes Curriculares, além de intimamente refletirem o projeto pedagógico e a vocação de cada IES, deverão ser profissionais dinâmicos, adaptáveis às demandas do mercado de trabalho, aptos a aprender a aprender, estando então diferenciados em relação àqueles formados no âmbito dos currículos mínimos estáticos.

As Diretrizes devem, então, fornecer as bases filosóficas, conceituais, políticas e metodológicas a partir das quais se define um conjunto de habilidades e competências, que configuram uma estruturação do conhecimento de uma certa área do saber. Devem ainda ser eixos estruturantes das experiências de aprendizagem, capacitando o aluno a lidar com o específico a partir de uma sólida base nos conceitos fundadores de sua área.

\section{CURSO: GEOLOGIA OU ENGENHARIA GEOLÓGICA}

\section{PERFIL DESEJADO DO EGRESSO}

As Diretrizes Curriculares devem possibilitar às IES definir diferentes perfis profissionais para cada área do conhecimento, garantindo uma flexibilidade de cursos e carreiras e promovendo a integração do ensino de graduação com a pós-graduação. Neste sentido, as IES devem contemplar, no perfil de seus formandos, as competências intelectuais que reflitam a heterogeneidade das demandas sociais em relação aos profissionais de alto nível, consoante à inovação presente no inciso II do artigo $43 \mathrm{da} \mathrm{LDB}$, que define como papel da educação superior o de "formar diplomados nas diferentes áreas de conhecimento, aptos para a inserção em setores profissionais" - Edital $N^{\circ}$ 4 de 10 de dezembro de 1997.

O Curso de Geologia deve formar um profissional com condição de trabalhar em qualquer área de atuação das Ciências Geológicas; que tenha interesse e capacidade para o trabalho de campo; visão abrangente das Geociências e de suas interações com ciências correlatas; pleno domínio da linguagem técnica geológica aliada à capacidade de adequação desta linguagem à comunicação com outros profissionais e com a sociedade; conhecimento de Ciências Exatas que permita abordagens quantitativas das informações geológicas; familiaridade com métodos e técnicas de informática, especialmente no tocante ao geoprocessamento. Para tanto, as Diretrizes Curriculares devem privilegiar nessa formação a capacidade de abordar e resolver problemas geológicos com competência, aliando uma sólida base teórica a um treinamento prático e intensivo. O egresso deverá ter atitude ética, autônoma, crítica, criativa, empreendedora e atuação propositiva, na busca de soluções de questões de interesse da Sociedade.

\section{COMPETÊNCIAS E HABILIDADES DO EGRESSO}

As Diretrizes Curriculares devem conferir uma maior autonomia às IES na definição dos currículos de seus cursos. Desta forma, ao invés do atual sistema de currículos mínimos, onde são detalhadas as disciplinas que devem compor cada curso, deve-se propor linhas gerais capazes de definir quais as competências e habilidades que se deseja desenvolver nos mesmos. Espera-se, assim, a organização de um modelo capaz de adaptar-se às dinâmicas condições de perfil profissional exigido pela sociedade, onde a graduação passa a ter um papel de formação inicial no processo contínuo de formação permanente, que é inerente ao mundo do trabalho - Edital $\mathrm{N}^{\circ} 4$ de 10 de dezembro de 1997.

No decorrer do curso o estudante deverá aprofundar sua formação para atender a qualquer uma das exigências do mercado de trabalho e da sociedade. Assim o Curso deve estabelecer, periodicamente, quais são essas exigências, tomando sempre o cuidado de não ficar estritamente atrelado ao mercado de trabalho. Para o momento atual, podem ser apontadas, entre outras, as seguintes competências:

1. Mapeamento geológico e as demais competências discriminadas na Lei 4076, de 23 de junho de 1962, tais como: trabalhos topográficos e geodésicos, levantamentos geoquímicos e geofísicos, estudos relativos as ciências da Terra, trabalhos de prospecção e pesquisa para a cubagem de jazidas e determinação de seu valor econômico, ensino de ciências geológicas, emitir parecer em assuntos legais relacionados com a especialidade, realizar perícias e arbitramentos referentes às matérias citadas; 
2. Planejar, executar, gerenciar, avaliar e fiscalizar projetos, serviços e ou pesquisas científicas básicas ou aplicadas que visem ao conhecimento e a utilização racional dos recursos naturais e do ambiente;

3. Pesquisar e otimizar o aproveitamento tecnológico dos recursos minerais e energéticos sob o enfoque de mínimo impacto ambiental;

4. Pesquisar novas alternativas de exploração, conservação e gerenciamento de recursos hídricos;

5. Fornecer as bases para o planejamento da ocupação urbana e para a previsão e prevenção de riscos de acidentes por desastres naturais e aqueles provocados pelo Homem;

6. Desenvolver métodos de ensino e pesquisa das Geociências voltados tanto para a melhoria do desempenho profissional como para a ampliação do conhecimento em geral;

7. Desenvolver e aplicar métodos e técnicas direcionadas a gestão ambiental;

8. Atuar em áreas de interface, como a Tecnologia Mineral, Ciências do Ambiente e Ciências do Solo.

\section{CONTEÚDO CURRICULAR}

O conteúdo curricular deve contemplar a formação básica e profissional do geólogo. Além disso, conteúdos temáticos adicionais poderão ser estabelecidos de acordo com as competências ou objetivos existentes nas Instituições de Ensino e inseridas no contexto regional de cada uma delas. Dividem-se, assim, os conteúdos em formação básica, formação geológica específica e formações temáticas, estas últimas estabelecidas segundo as características e competências de cada Curso.

\section{Conteúdo Básico}

O conteúdo de formação básica deverá possuir caráter obrigatório. Propõe-se a seguinte composição para a formação básica em Geologia:

Conteúdos em Matemática, Estatística, Física, Computação, Química, Biologia e Geociências.

\section{Conteúdo para a formação geológica específica}

O conteúdo para a formação geológica específica deverá também possuir caráter obrigatório e abranger tópicos considerados indispensáveis à formação do geólogo. Propõe-se a seguinte composição para o conteúdo de formação específica em Geologia:

Conteúdos em Mineralogia, Cristalografia, Topografia, Petrologia, Petrografia, Sedimentologia, Paleontologia, Geologia Estrutural, Geotectônica,
Estratigrafia, Geoquímica, Geofísica, Geologia Histórica, Geologia do Brasil, Fotogeologia, Sensoriamento Remoto, Pedologia, Geomorfologia, Geologia Econômica, Prospecção, Mapeamento Geológico, Recursos Hídricos e Recursos Energéticos.

\section{Conteúdos Temáticos}

Os temas específicos terão um caráter optativo podendo ser permitido ao aluno mesclar vários tópicos entre aqueles oferecidos por cada Curso. Pretende-se, desta forma, dotar o aluno de uma formação polivalente e adequar as Instituições de Ensino às realidades e demandas regionais. Propõemse os seguintes conteúdos temáticos, ficando as IES com liberdade para adaptá-los conforme as suas características, ou criar outros, caso julguem necessários:

Geoprocessamento;

Recursos Minerais;

Recursos Energéticos;

Recursos Hídricos;

Geologia de Engenharia;

Geologia Ambiental;

Geologia Regional;

Geofísica Aplicada;

Licenciatura em Geociências;

\section{Conteúdo Complementar}

O conteúdo complementar é de caráter optativo, buscando oferecer ao aluno, a critério de cada IES, conteúdos, entre outros, de Ciências Humanas, tais como: Economia, Filosofia da Ciência, Gestão, Administração e Comunicação.

\section{TRABALHO DE CONCLUSÃO DE CURSO}

Para integralização do Curso de Geologia ou Engenharia Geológica, o aluno deverá elaborar um trabalho de conclusão de curso, sobre temas de conteúdo geológico.

\section{DURAÇÃO DO CURSO}

A duração proposta para o curso, adotada no currículo a ser oferecido pela IES, é de cinco anos no mínimo e seis anos no máximo, com tempo médio recomendado de cinco anos, ou dez semestres. A carga horária mínima é de 3600 horas.

\section{ATIVIDADES DE CAMPO}

Deve ser planejada e incluída na programação dos cursos uma carga horária mínima de atividades de campo, que pode estar contida, ou não, na carga 
horária de diferentes disciplinas; o nível mínimo requerido é de $20 \%$ do total do curso (por exemplo, 720 horas para um curso de 3600 horas).

\section{ESTÁGIO SUPERVISIONADO}

É desejável a inclusão, no leque de disciplinas de cursos de graduação em Geologia, de uma disciplina (ou atividade) denominada Estágio Supervisionado, que proporcionará ao aluno conhecimentos e experiência profissionais, ao permitir a interação da escola com a empresa. A disciplina (ou atividade) pode ser obrigatória ou não. A carga horária mínima sugerida, na empresa, é de 120 horas a 160 horas, podendo ser concentrada em intervalo de 30 dias, no recesso de aulas escolares, ou eventualmente dispersa no semestre.

\section{INTERCÂMBIO INTERINSTITUCIONAL}

A adoção de facilidades para intercâmbio de alunos na parte final dos Cursos de Geologia, entre instituições, para cumprir atividades ou disciplinas de ênfases inexistentes nas unidades de origem pode propiciar o aprimoramento dos futuros profissionais. Recomenda-se que as IES busquem proporcionar tal intercâmbio entre os diferentes cursos, para desenvolver conteúdos e adquirir habilitações nas áreas temáticas, de acordo com as regras específicas das diferentes universidades.

\section{ESTRUTURA CURRICULAR}

A estrutura do curso deverá ser caracterizada pela distribuição equilibrada entre disciplinas de conteúdo obrigatório e aquelas optativas escolhidas segundo o conteúdo temático correspondente.

As disciplinas obrigatórias ficarão restritas aos conteúdos básicos e de formação geológica específica. $\mathrm{O}$ aluno poderá escolher disciplinas optativas que compõem os conteúdos temáticos ou mesmo mesclar conteúdos entre os vários oferecidos.

\section{ATIVIDADES COMPLEMENTARES}

Deve ser estimulada a participação do aluno em atividades extra-curriculares, tais como: iniciação científica, projetos de pesquisa, monitoria didática ou estágios em áreas relacionadas à Geologia ou suas interfaces.

Preferencialmente no último ano do Curso, a IES deverá estimular o aluno a desenvolver atividades de estágios em instituições externas à Universidade, sob a supervisão de um professor, apresentando um relatório técnico de suas atividades.

Subscrevem o presente documento os participantes do VII Encontro do Fórum Nacional de Cursos de Geologia:

Lúcia Maria Fantinel (Presidente); Valdecir de Assis Janasi; José Fernando Pina Assis; Celso Dal Ré Carneiro; Beatriz Paschoal Duarte; Carlos Humberto da Silva; Emilio Velloso; Fernando Mancini; Gérson Fauth; Harrizon Lima de Almeida; José Fernando Pina Assis; José Roberto Alecrim; Maurício Compiani; Messias Menezes; Paulo Fortes; Rommulo Conceição; Vanildo Pereira da Fonseca; Washington B. Leite Júnior; Wellington Silva Filho.

\section{Referências Bibliográficas}

Carneiro C.D.R. 2005. Relato Final do II Seminário Nacional sobre Cursos de Graduação em Geologia. Campinas, abril de 2002. Terre Didatica, 1(1):55-63. URL: < http://www.ige.unicamp.br/ terraedidatica/ $>$

Mesquita F.J.G., Artur A.C., Lazzarotto A., Misi A., Leipnitz B., Barros C.E., Carneiro C.D.R., Tubbs Filho D., Assis F.P., Abreu F.A.M., Sobreira F., Moura M.A., Toledo M.C.M., Souza M.A.T.A.de, Costa R.D., Zouain R.N.A., Menegat R., Nadalin R.J., Santos R.A.A.dos, Vasconcelos S.M.S., Marques T.M., Neri T.F.O., Dias V.M., Souza Z.S.de. 2001. Sugestões de Alteração para a Proposta de Diretrizes Curriculares para os Cursos de Graduação em Geologia e Engenharia Geológica. Salvador: Inst. Geoc. UFBA. 9p. (inédito, baseado nas contribuições do I Seminário Nacional sobre Cursos de Graduação em Geologia, Salvador, 30.05 a 01.06.2001).

Nummer A.R., Godoy A.M., Lazzarotto A., Carneiro C.D.R., Schultz C.L., Tubbs Filho D., Guimarães E.M., Althoff F., Assis J.F.P., Pinho F.E.C., Sobreira F., Carvalho I.S., Sabadia J.A.B., Fernandes Filho L.A., Toledo M.C.M.de, Fernandes M.L.S., Costa R.D.da, Machado R., Menegat R., Nadalin R.J., Santos R.A.A.dos, Vasconcelos S.M.S., Marques T.M., Souza Z.S.de. 2005. Diretrizes Curriculares para os Cursos de Graduação em Geologia e Engenharia Geológica. Terre Didatica 1(1):64-69. URL: < http://www.ige.unicamp.br/terraedidatica/ v1n1/t didatica 2005 v01n01_p064-069 nummer_et_al.pdf $>$.

Sobreira F. 2005. Relato Final do I Seminário Nacional sobre Cursos de Graduação em Geologia. Salvador, maio de 2001. Terra Didatica, 1(1):51-54. URL: $<$ http://www.ige.unicamp.br/terraedidatica/ $>$. 